\title{
À propos de deux ouvrages de Bernard Juillerat
}

\section{Gilles Bounoure}

\section{(2) OpenEdition}

Journals

Édition électronique

URL : http://journals.openedition.org/jso/6056

DOI : 10.4000/jso.6056

ISSN : 1760-7256

\section{Éditeur}

Société des océanistes

\section{Édition imprimée}

Date de publication : 15 décembre 2010

Pagination : 139-144

ISBN : 978-2-85430-027-7

ISSN : 0300-953x

Référence électronique

Gilles Bounoure, «À propos de deux ouvrages de Bernard Juillerat », Journal de la Société des

Océanistes [En ligne], 130-131 | 2010, mis en ligne le 15 décembre 2013, consulté le 11 juin 2020. URL: http://journals.openedition.org/jso/6056 ; DOI : https://doi.org/10.4000/jso.6056 


\section{À propos de deux ouvrages de Bernard Juillerat}

par

Gilles BOUNOURE*

Derniers volumes de Bernard Juillerat parus de son vivant, Penser l'imaginaire (2001) et Psychanalyse et anthropologie (2005, avec Patrice Bidou et Jacques Galinier pour co-éditeurs) n'ont jamais été décrits dans le JSo. Comme ils sont relativement récents et qu'ils semblent, séparément mais surtout ensemble, susceptibles de rendre sensibles certaines des conceptions de l'anthropologie qu'avait élaborées cet éminent chercheur au sommet de sa carrière et de ses réflexions, il a paru utile de relever ici quelquesunes de leurs propositions essentielles, en complément de l'hommage qui lui est rendu dans ce numéro.

L'un et l'autre de ces volumes sont des recueils d'articles, extraits pour la plupart de travaux antérieurs dans les dix chapitres du premier, contributions inédites dues à neuf auteurs pour le second. Cette similitude de forme doit certainement moins aux contingences de l'édition scientifique qu'aux méthodes de travail et à la manière de pratiquer l'anthropologie de B. Juillerat, par « rencontres et divergences »-c'est le titre de la première partie de Penser l'imaginaire -, convergences et "confrontations", qu'attestent encore les nombreux ouvrages collectifs dont il fut le maître d'œuvre ou l'un des principaux concepteurs. À côté de l'évidence que «l'anthropologie ne peut être que pluridisciplinaire» (Penser l'imaginaire, p. 5), il n'est pas exclu non plus qu'une telle forme soit nécessaire, au moins dans les circonstances actuelles, aux progrès de «l'anthropologie psychanalytique », sujet commun de ces deux publications.

Penser l'imaginaire, dont André Green souligne dans le présent numéro le caractère magistral et l'importance décisive du point de vue de l'ethnopsychanalyse, ne fait pas que résumer les tra- vaux antérieurs d'un « ethnologue revendiquant le recours à la psychanalyse freudienne pour l'interprétation des représentations culturelles » (p. 111), il en montre l'actualité pressante, à l'orée du XxI ${ }^{\mathrm{e}}$ siècle. Y relire (chap. 10, «À propos de Yangis ", pp. 247-271) des extraits de L'Avènement du père (1995, chap. 2, 4 et 6, avec modifications de détail et «repentirs» nombreux, comparer par exemple le texte de 2001, pp. 247-248 et celui de 1995, pp. 67-69) conduit à reconsidérer la portée de cette monographie parue d'abord dans une collection s'adressant en apparence surtout aux spécialistes (« chemins de l'ethnologie ») et dont l'écho pouvait difficilement dépasser ce petit cercle. Il aura fallu lire toute la première partie de Penser l'imaginaire pour que se révèlent pleinement les enjeux généraux (généreux aussi bien) de cette « confrontation avec le terrain » (titre de la seconde partie du volume).

Mais la première partie de ce livre, constituant les quatre septièmes du corps du texte, n'est-elle pas trop théorique, générale et exagérément éloignée des questions concrètes qui se posent à beaucoup de scientifiques spécialistes de l'Océanie? Pourquoi se préoccuper de "la dérive cognitiviste en anthropologie » (introduction inédite), du dialogue malaisé entre anthropologie et psychanalyse (chap. 1), de la question des universaux (chap. 2), de "la dissidence jungienne » (chap. 3), de "l'atome de parenté » (chap. 4) et de la mythologie matriarcale de Bachofen (chap. 5) ? Là encore, ces textes, où il y a beaucoup à apprendre sur des débats importants dont B. Juillerat était remarquablement informé, mais qui étaient parus à plusieurs années de distance (de 1988 à 2001), prennent un autre sens à se trouver réunis comme autant

* flongue@wanadoo.fr 
d'étapes d'une réflexion toute tendue à rendre sa place à l'imaginaire dans l'analyse et la compréhension des sociétés humaines, direction qu'assignait plus récemment Maurice Godelier aux recherches anthropologiques (Au fondement des sociétés humaines, 2007, voir JSo 126-127).

B. Juillerat n'aura certes pas été le premier à faire intervenir la psychanalyse dans des travaux d'ethnologie, ou plutôt à vouloir la "mettre à l'épreuve de faits ethnographiques ", et il rapporte brièvement (p. 45 et suiv.) les efforts de ses prédécesseurs, non sans raccourcis approximatifs quelquefois. Par exemple, Rivers et Seligman, qui avaient leur carrière derrière eux quand ils ont découvert Freud grâce à Jones, ont été empêchés d'en tirer parti dans le domaine de l'ethnologie mélanésienne non par «l'héritage d'une psychologie expérimentale » qui aurait limité leur intérêt pour la psychanalyse, mais par la Grande Guerre, et les soins qu'ils donnaient aux combattants victimes du " shell shock», au moment même où ils faisaient cette découverte. Rivers est mort peu après, mais dans la décennie suivante, après avoir déclaré devant le Royal Anthropological Institute, en 1924, que les mécanismes psychiques fondamentaux des nonEuropéens, même sauvages ou barbares s'avèrent "les mêmes que les nôtres", Seligman a suscité des recherches pionnières sur le rêve, en Amérique du Nord aussi bien qu'en Mélanésie (lire aussi p. 48 et 104, Gilbert et non " Gérard Durand ", auteur assez connu en son temps des Structures anthropologiques de l'imaginaire, 1960, absent de la bibliographie, etc.). Ces inexactitudes n'ont heureusement pas de caractère déterminant dans l'économie générale de l'ouvrage, décisif quant à lui.

S'il doit susciter un tel qualificatif, c'est qu'il cherche - et réussit - à définir de façon remarquablement précise ce que peut être, et ce que doit devenir «le recours à la psychanalyse freudienne » en ethnologie, et plus largement dans les « sciences humaines et sociales ». Même chez les psychanalystes freudiens, la validité d'un tel recours ne fait pas l'unanimité, et Olivier Flournoy (1979 : 120-121) était fondé à écrire :

« Les ethnologues qui lient l'exogamie au complexe d'Edipe devraient toujours se souvenir que ce dernier est un modèle explicatif d'une expérience qui se déroule dans un temps particulier, et non d'un comportement humain. Le psychanalyste n'est pas le détenteur, ni Freud le fondateur, d'une vérité universelle. L'expérience psychanalytique est intersubjective. L'interprétation métapsychologique est la mise en commun de cette expérience par reconstitution d'une histoire originale de l'analysé. Et la théorie psychanalytique va faire le pont entre les membres de la communauté analytique et ceux de la communauté univer- selle. De ce fait, l'analyse ne saurait échapper aux contradictions du monde, et la vérité intime de l'expérience, celle à deux de la métapsychologie, risque par là de devenir vérités dogmatiques de la théorie analytique, si cette dernière s'adresse à tous en omettant de préciser ses origines. »

Sans citer cet auteur (qui a évoqué par ailleurs la nécessité pour tout analyste de «théoriser »), B. Juillerat ne méconnaît pas les arguments des partisans d'une psychanalyse avant tout « clinique » ni, à l'inverse, les risques d'une « ethnographie clinique ", psychanalyse de terrain peu cohérente parce que mal assumée (p. 50). Mais les obstacles principaux ont cessé de venir des tendances dogmatiques ou irresponsables qui caractérisaient naguère certaines " écoles 》 psychanalytiques, aujourd'hui dépassées par un renouveau des investigations devant beaucoup à des chercheurs d'esprit libre et rigoureux comme André Green. Ils ne viennent pas non plus des anthropologues qui ont discuté sans concession les travaux de B. Juillerat, à l'instar d'Alfred Gell écrivant dans Shooting the sun (1992 :141-2) :

«I am skeptikal of the idea that actual desire to commit incest with the mother is a genuine motive, even unconsciouly, as far as Umeda males are concerned. The representation of the mother throughout Melanesia is monotonously geared to the provision of food, not so much milk as vegetable staples. Why import a complex of ideas about incest with the mother, historically associated with the development of our own system of family roles, childcare practices, sexual stereotypes, or ideas about purity into such an alien context $[\ldots]$ ? »

Car voilà non seulement des arguments anciens qui appellent des réponses qu'on pourrait également dire "classiques », et qui semblent d'ailleurs avoir de moins en moins de partisans même chez les tenants de l'existence de sociétés " non œdipiennes », mais voilà surtout des idées toujours susceptibles d'être débattues entre anthropologues et psychanalystes, dont " chacune [des disciplines] a aidé aux progrès de l'autre ", comme le remarquait déjà Sacha Nacht il y a plus de quarante ans (1968: 1704). Tel n'est plus le cas avec le cognitivisme.

Comme l'indique B. Juillerat, le danger qu'il représente pour l'anthropologie tient à son dogmatisme scientiste, incapable de prendre en compte l'imaginaire, et à ses prétentions à l'hégémonie dans les sciences humaines et sociales, sans dialogue possible :

« La plupart des anthropologues se réclamant du cognitivisme partent d'une théorie mal assurée, puis l'illustrent a posteriori par des exemples ethnographiques ad hoc qui n'ont alors qu'une fonction pédagogi- 
que [...]. La méthode scientifique ne consiste-t-elle pas plutôt à partir du particulier et de la comparaison pour en tirer des règles générales ?» (p. 38)

À propos des cognitivistes, Marc Richelle (1993 : 25-27) avait montré auparavant combien :

« les hypothèses de travail sur [les] rapports [de la conscience] à la machine nerveuse demeurent, pour l'essentiel, ce qu'elles ont été à travers les $\mathrm{XIX}^{\mathrm{e}}$ et $\mathrm{XX}^{\mathrm{e}}$ siècles : pour les uns, monistes et matérialistes, plus on explorera les données physiques - cerveau, neurones, et aussi, pourquoi pas, comportements - plus on approchera d'une émancipation définitive par rapport aux notions immatérielles d'esprit, d'âme, etc. ; pour les autres, le fait que l'accumulation des progrès en ce domaine - en cette matière - ne semble pas nous aider à résoudre le problème central apparaît désarmant, et justifie la fidélité ou le retour au dualisme spiritualiste. Chacun vient encore en neurosciences avec ses préférences, venues d'ailleurs. »

Pourtant, signalait-il aussi, cette « accumulation des progrès » encourageait déjà une position "clairement réductionniste», affirmant «que les conduites se laissent décrire en termes purement neuronaux. Il faut entendre, sans aucun doute, que les autres descriptions sont ou bien inadéquates ou bien provisoires - l'idée que les descriptions exclusivement neuronales sont impossibles n'est pas envisagée ». Aujourd'hui comme hier, l'assurance empruntée des cognitivistes - elle n'est pas sans évoquer certains procédés incriminés dans l'actuelle crise financière mondiale, tel le recours aveugle aux modélisations mathématiques, et procède même d'une sorte de « foi » scientiste, selon B. Juillerat (p. 15) - ne leur vient que des sciences "dures» auxquelles ils se réfèrent, parfois hégémoniques elles aussi malgré l'étroitesse de leurs champs d'application. Parmi elles, du fait des intérêts, économiques et politiques notamment, qui leur sont associés, les «neurosciences » ainsi que les techniques et les molécules qu'elles permettent d'expérimenter donnent lieu à de graves « dérives ", recours aux psychostimulants ou aux bétabloquants, expériences de "neuromarketing », etc. Hervé Chneiweiss, éminent neurobiologiste lui-même, en a dressé il y a peu (2006) un impressionnant catalogue, valant rappel aux règles scientifiques autant qu'aux valeurs morales, civiques et humanistes en principe attachées à ce type de recherche.

Il est manifeste, d'après ce dernier ouvrage, que ces " dérives » (telle l'utilisation de la Ritaline "hors de toute raison médicale», p. 68, récemment proposée en France) menacent souvent « l'autonomie de la pensée, la vie privée, et l'exercice de la liberté » individuelle ou collective (p. 61), et dessinent sans le dire des projets de sociétés peu vivables, au-delà du "contrôle social des passions » (p. 57) auquel s'emploient déjà maintes multinationales de la pharmacie, de la distribution et du divertissement ainsi que nombre d'agences gouvernementales de sécurité. On pourrait en dire autant du cognitivisme appliqué, des "sciences de l'information » intégrées aux "outils de gestion des ressources humaines ", ou des nouvelles formations universitaires en «cognition naturelle et artificielle» visant par exemple à améliorer « les interactions humain-machine » en " psycho-informatique » et ailleurs, ainsi que le suggéraient déjà d'anciens travaux cognitivistes assimilant la mémoire humaine aux capacités de stockage des ordinateurs (par exemple Lindsay et Norman, 1980 : 302-332). Si « comme l'écrit André Green, "on a l'impression que les cognitivistes vivent dans un monde créé par eux..." " (Penser l'imaginaire, p. 27), on peut également craindre à bon droit qu'ils ne cherchent à imposer leur monde aux autres, notamment par le biais d'une " anthropologie » cognitiviste, supposé qu'on puisse réunir ces termes tellement antithétiques.

Ainsi, mieux que le behaviorisme qu'il prolonge sous la bannière plus neuve des neurosciences, le cognitivisme reproduit et tend à imposer « une façon de penser l'homme typiquement nord-américaine, inspirée de la cybernétique et de l'intelligence artificielle, attisée par le développement de l' "information" " (p. 12, à rapprocher $\mathrm{du}$ 《syndrome très américain » décrit p. 67sq. par H. Chneiweiss comme sous-jacent aux dérives qu'il énumère). Ces enjeux généraux ne peuvent être oubliés, ce que B. Juillerat ne manque pas de rappeler brièvement mais fermement. Il se garde néanmoins, en toute rigueur, de porter ses critiques de "la dérive cognitiviste » hors du champ de l'anthropologie, où éclate déjà, sans la moindre ambiguïté, le caractère mutilant de «cette vision mécaniste » qui ignore l'imaginaire :

«Cette vision est doublement réductrice: d'une part parce qu'elle ne prend pour objet d'étude que les capacités cognitives de l'homme, d'autre part parce qu'elle réduit la cognition elle-même à la formation et à la transmission d'unités ou de systèmes catégoriels. Or la culture n'est pas seulement du cognitif (stricto sensu) et le cognitif pas seulement du catégoriel. » (Penser l'imaginaire, pp. 12 et 23)

Le même souci de porter le débat au plus haut anime le texte que consacre André Green, dans le volume édité par Patrice Bidou, Jacques Galinier et B. Juillerat, au " psychisme entre biologie et 
anthropologie » (pp. 27-34). Il n'y est plus question de l'idéologie cognitiviste, mais des recherches mêmes dont elle se réclame et dont A. Green est bon connaisseur, comme médecin formé à la psychiatrie. Il y a d'un côté « l'universalité biologique forcément réductrice », de l'autre « la diversité phénoménale de l'anthropologie », le passage de l'une à l'autre ne pouvant s'expliquer par une causalité exclusivement « neuronale », la seule qu'envisagent jusqu'à présent les neurobiologistes. B. Juillerat écrivait antérieurement :

« C'est toute la question de la causalité qui est mal comprise par le cognitivisme. André Green a proposé de prendre en compte une 'causalité psychique' qui s'inscrit pour nous, de façon évidente, dans la recherche d'explication des phénomènes culturels. »

Et il formulait « l'idée », difficilement contestable :

« que l'élaboration de la culture relève d'un cumul, mais aussi d'un conflit des logiques et des significations, donc d'une pluralité des causes. » (Penser l'imaginaire, pp. 37-38)

La «pluralité des causes » définit le principe scientifique (ou même analytique dans le sens originel du terme) qui permet de préciser les ambitions et les limites du « recours à la psychanalyse freudienne pour l'interprétation des représentations culturelles ». "L'anthropologie ne saurait faire à la psychanalyse qu'une place limitée, mais [...] essentielle» dictée non par «l'alternative pour ou contre Freud » mais par des questions générales portant sur le rôle du « fonctionnement psychique, dans ses contenus et ses mécanismes ", inconscients comme conscients, dans "l'élaboration culturelle » des sociétés. Si l'on peut attendre le meilleur des "possibilités de coopération insuffisamment explorées » à ce jour entre ces disciplines, « cela ne signifie aucunement qu'elles puissent prétendre à un discours unitaire confondant le sujet individuel et sa culture, la diversité des cultures avec celle des subjectivités, ou encore le psychogénétique avec le sociogénétique. 》 " Il s'agit au contraire de s'opposer à tous les réductionnismes et de s'orienter vers une complémentarité méthodologique non éclectique mais sélective par souci d'adéquation à l'objet, donc de vérité. » (ibid. : 7, 53-54).

Tout autant que Penser l'imaginaire, les " regards croisés » réunis dans Anthropologie et psychanalyse apportent des preuves consistantes de la validité et de la fertilité d'une telle démarche, heureusement suivie par des spécialistes d'autres aires culturelles, les américanistes Patrice Bidou et Jacques Galinier en premier lieu. La plupart des contributions sont de grand intérêt, mais pour s'en tenir à l'Océanie, celle de Gillian Gillison, « Totem et tabou dans les Hautes Terres de Papouasie Nouvelle-Guinée. La révolte des filles » (pp. 99-124), mérite certainement d'être lue et méditée, pour tout ce qu'elle révèle d'inédit des mœurs et conceptions des Gimi, mais aussi pour les nombreux parallèles qu'elle suggère avec d'autres sociétés mélanésiennes, rôle des plates-formes, rêveries autour du serpent et du pénis géant, etc. Il est également significatif de la même démarche collective, pluridisciplinaire et non pas seulement " complémentariste » mais « dialogique » (pp. 8-9), que la présentation analytique de cette contribution et de celle de B. Juillerat ait été rédigée par P. Bidou, dans l'introduction à trois voix successives ouvrant le volume, tandis que B. Juillerat y commentait d'autres articles pour désigner en conclusion (p. 24) comme autant « de signaux alarmants [...] l'avancée de la mondialisation, l'uniformisation des genres de vie et de pensée, la diffusion épidémique du New Age », s'ajoutant aux tentatives de mainmise " cognitiviste » sur l'ethnologie et les sciences sociales qu'il évoquait aussi. Cela le conduisait à parler de «course contre la montre » pour les anthropologues, en suggérant qu'elle concerne beaucoup d'autres que ces seuls spécialistes, évidence peut-être un peu mieux admise aujourd'hui qu'en 2005.

La contribution principale de $\mathrm{B}$. Juillerat à ce deuxième volume ( Plaisir et réalité. La notion de "cargo" revisitée », pp. 81-97) répond implicitement au reproche fait de longue date aux tenants de l'interprétation psychanalytique de ne pouvoir envisager les sociétés dans leurs dimensions historiques (voir Penser l'imaginaire, par exemple pp. 52-53). Une telle critique ne pouvait atteindre l'auteur de la remarquable enquête sur Thurnwald et les Banaro, s'appuyant précisément sur les transformations diachroniques de cette société pour montrer comment Thurnwald l'avait "modélisée », ramenée à " une sorte de "modèle réduit" " (La révocation des Tambaran, p. 177), à ranger parmi d'autres inventions malvenues $\mathrm{du}$ discours anthropologique. $\mathrm{Ne}$ pourrait-on en dire autant des " données ethnologiques " aujourd'hui disponibles sur les mouvements de type " cargo » au $\mathrm{Xx}^{\mathrm{e}}$ siècle, forcément incomplètes et réductrices du fait du contexte colonial et répressif dans lequel se développèrent ces mouvements et furent menées les premières enquêtes à leur sujet ? Il est en tout cas hors de doute qu'en Mélanésie ces réactions à la profusion subite de marchandises occidentales 《 à ne toucher que des yeux » furent « tantôt mal comprises par les missionnaires et considérées 
comme menaçant l'ordre public par les administrateurs, tantôt sous-estimées dans leur dimension psychique par certains anthropologues au profit d'explications plus matérialistes ou plus politiques, tantôt réduites à des troubles mentaux par certains ethnopsychiatres. » (p. 82).

N'envisageant, faute de place, « qu'un exemple situé dans l'espace et dans le temps historique », B. Juillerat revient sur « le culte du cargo » qu'il a « eu l'occasion d'observer chez les Yafar de Papouasie Nouvelle-Guinée en 1981 », dans un climat d'escarmouches, d'intrusions et parfois de massacres à la frontière de l'Irian Jaya, également propice à des attentes de bouleversements de l'ordre quotidien. "Travail ou fertilité » sans effort, l'actualité rappelait incessamment cette alternative. L'article montre par une analyse très serrée ce qui, " de la pulsion de savoir » et de partager le secret de cette fertilité, a conduit « aux représentations cultuelles de 1981 et à [leurs] corrélats mythiques ». On ne sait ce qu'il faut admirer le plus, de l'élégante concision de la démonstration ou de l'équilibre impeccable de la conclusion. Faute de pouvoir résumer ici la première, il semble indispensable, pour finir, de citer la seconde, parfaite définition du principe de «pluralité des causes» devant présider au « recours à la psychanalyse freudienne pour l'interprétation des représentations culturelles », selon les termes mêmes de B. Juillerat :

« Les anthropologues ayant étudié la question des cultes millénaristes de Mélanésie se sont presque toujours distancés de Freud ; certains ont formulé ce rejet clairement sans tenter de mettre la notion de cargo ou de salut social à l'épreuve de la psychanalyse. Ce que nous avons essayé ici ne doit pas être vu comme un réductionnisme, mais seulement comme l'éclairage d'un aspect jusque-là négligé du fantasme d'appropriation du cargo. Il fallait pour cela prendre en compte la causalité interne, inconsciente, et non seulement les circonstances historiques et les motivations rationnelles du culte. Ces deux ordres de facteurs se complètent ; alors que le second est déterminé par les événements factuels extérieurs et les motivations conscientes, le premier fournit le contenu fantasmatique et met en œuvre les mécanismes psychiques socialisés tout en puisant dans le corpus symbolique déjà existant de la culture. Expliquer un champ sans l'autre équivaudrait à amputer la réalité globale. » (p. 97)

Ce dernier article ne fait pas seulement regretter que B. Juillerat ait été empêché de donner des suites à cet «éclairage » exceptionnellement lumineux, il fait souhaiter de le voir réuni avec d'autres, s'il est possible, dans un volume qui prolongerait Penser l'imaginaire.

\section{BIBLIOGRAPHIE}

ChNeIweIss Hervé, 2006. Neurosciences et neuroéthique. Des cerveaux libres et heureux, Paris, Alvik.

Flournoy Olivier, 1979. Le temps d'une psychanalyse, Paris, Belfond.

JuILlerat Bernard (ed.), 1992. Shooting the sun. Ritual and Meaning in West Sepik, Washington, Smithsonian Institution Press.

JuILleRAT Bernard, 1993. La révocation des Tambaran. Les Banaro de Richard Thurnwald revisités, Paris, CNRS Éditions.

- 1995. L'avènement du père. Rite, représentation, fantasme dans un culte mélanésien, Paris, CNRs Éditions - Éditions de la maison des sciences de l'homme.

_, 2001. Penser l'imaginaire. Essais d'anthropologie psychanalytique, Lausanne, Éditions Payot (bibliographie, triple index), $320 \mathrm{p}$.

Juillerat Bernard, Patrice BIDOu et Jacques GALINIER (s.d.), 2005. Psychanalyse et anthropologie. Regards croisés, Paris, Éditions de l'EHEss, Cahiers de l'Homme, $230 \mathrm{p}$.

Lindsay Peter H. et Donald A. Norman, 1980. Traitement de l'information et comportement humain. Une introduction à la psychologie, Montréal-Paris, Études vivantes (traduction de l'édition américaine de 1977).

NACHT Sacha, 1968. "Psychanalyse et ethnologie », in Jean Poirier (éd.), Ethnologie générale, Paris, Gallimard, Encyclopédie de la Pléiade, pp. 1680-1705.

Richelle Marc, 1993. Du nouveau sur l'esprit, Paris, Presses universitaires de France. 
